\title{
Assessment of the risks of a myasthenic crisis after thymectomy in patients with myasthenia gravis: a systematic review and meta-analysis of 25 studies
}

\author{
Chaoying Liu, Peng Liu, Xiao jing Zhang, Wen qian Li and Guoyan Qi
}

\begin{abstract}
Background: Despite the burgeoning literature describing preoperative and postoperative risks of a myasthenic crisis after thymectomy (MCAT) in patients with myasthenia gravis, substantial differences exist in the risk factors identified by previous studies. We conducted a meta-analysis to assess the reported risk factors and MCAT risk.

Methods: We collected relevant studies on the risk factors for MCAT by searching the PubMed, Embase, The Cochrane Library, China Biology Medicine (CBM), WanFang Data, VIP and CNKI databases. The search period ranged from the establishment of the database to November 2019.

Results: Twenty-five of the 458 identified studies were eligible for the meta-analysis. Seven retrospective cohort studies and 18 case-control studies were included, and 14 risk factors for MCAT were extracted. Meta-analyses of the association between MCAT and risk factors related to the patient's preoperative condition included a preoperative history of $M C$, preoperative bulbar symptoms, $\|\mathrm{la}+\| \mathrm{lb}+\mathrm{II}+\mathrm{VI}, \| \mathrm{l} \mathrm{l}+\mathrm{II}+\mathrm{VI}, \mathrm{VI}+\mathrm{V}$, dosage of pyridostigmine bromide prior to the operation, a preoperative AchR-Ab level $>100(\mathrm{~nm} / \mathrm{L})$, preoperative pulmonary function, preoperative complications, and preoperative disease course. Meta-analyses of the association between MCAT and surgery-related risk factors included intraoperative blood loss $>1000 \mathrm{~mL}$ and the mode of operation. Meta-analyses of the association between MCAT and postoperative risk factors included postoperative lung infection, thymoma and the WHO classification. The operation time was not an independent risk factor for MCAT.

Conclusions: The independent risk factors for MCAT were a preoperative history of MC, preoperative bulbar symptoms, preoperative MG Osserman stage, preoperative dosage of pyridostigmine bromide, preoperative serum AchR-Ab level, lung function, major postoperative complications, disease duration before thymectomy, blood loss, thoracotomy, postoperative lung infection, thymoma, and WHO classification.
\end{abstract}

Keywords: Myasthenic crisis, Thymectomy, Myasthenia gravis, Meta-analysis, Risks

*Correspondence: zzjwlsys@163.com

Hebei Key Laboratory of Myasthenia Gravis, Center of Treatment of Myasthenia Gravis Hebei Province, First Hospital of Shijiazhuang, No. 9

Fangbei Road, Chang'an District, Shijiazhuang 050011, Hebei Province, China

(c) The Author(s). 2020 Open Access This article is licensed under a Creative Commons Attribution 4.0 International License, which permits use, sharing, adaptation, distribution and reproduction in any medium or format, as long as you give appropriate credit to the original author(s) and the source, provide a link to the Creative Commons licence, and indicate if changes were made. The images or other third party material in this article are included in the article's Creative Commons licence, unless indicated otherwise in a credit line to the material. If material is not included in the article's Creative Commons licence and your intended use is not permitted by statutory regulation or exceeds the permitted use, you will need to obtain permission directly from the copyright holder. To view a copy of this licence, visit http://creativecommons.org/licenses/by/4.0/ The Creative Commons Public Domain Dedication waiver (http://creativecommons.org/publicdomain/zero/1.0/) applies to the data made available in this article, unless otherwise stated in a credit line to the data. 


\section{Introduction}

Myasthenia gravis (MG) is an autoimmune disorder induced by neurotransmission defects at the neuromuscular junction and is characterized by muscle weakness and fatigue [1]. MG is typically treated with anticholinesterase agents, surgical thymectomy, or immunosuppression and with short-term immunotherapies (plasmapheresis and intravenous immunoglobulin administration) to reduce the risk of exacerbations [2]. Thymectomy has been selected as a surgical treatment for patients with MG since Blalock reported its efficacy as a treatment for MG complicated with or without thymoma [3, 4]. However, 3-30\% of patients with MG still develop a myasthenic crisis after thymectomy (MCAT) [5-8]. An MG crisis (MC) represents a serious, life-threatening, rapid worsening of $\mathrm{MG}$ and potential airway compromise due to ventilatory or bulbar dysfunction [9]. MC is the main cause of death after MG thymectomy. Many risk factors for MCAT have been reported, including internal factors and surgical factors, such as a myasthenic crisis, medulla oblongata muscle weakness and operation time. Geng et al. [10] performed a meta-analysis of risk factors for a myasthenic crisis after thymectomy that included 15 trials and 2626 patients with MG. However, substantial differences exist in the risk factors for MCAT identified by previous studies. The included studies exhibited substantial heterogeneity, and the previous meta-analysis [10] did not provide any details in their methods section on their plan to address the heterogeneity. The aetiology of MCAT remains unclear, and an effective risk assessment system for predicting the occurrence of MCAT is not available. Therefore, we conducted a meta-analysis to assess reported risk factors and the risk of MCAT and to achieve an adequate sample size required for a comprehensive and precise estimation of the associations between these factors and MCAT.

\section{Methods}

\section{Literature sources and search strategy}

We collected relevant studies examining the risk factors for MCAT by searching the PubMed, Embase, The Cochrane Library, CBM, WanFang Data, VIP and CNKI databases. The search period extended from the establishment of the database to November 2019.

The search was conducted using a combination of subject words and free words. We used the following search terms: (myasthenic crisis) AND ("thymectomy [MeSH Terms]" OR "thymectomies").

We also used combinations of the following search terms to expand the search: (myasthenic crisis) AND ("risk factors [MeSH Terms]" OR "factor", risk" OR "risk factor" OR "population* at risk" OR "risk, population* at").

\section{Inclusion criteria}

The following inclusion criteria were used: (1) Patients with MG after thymectomy; (2) Intervention: provides risk factors for patients with MG after thymectomy; (3) Comparator: division of participants into a case group and a control group according to the occurrence of a myasthenic crisis; (4) Outcome: risk of MCAT reported as an adjusted odds ratio (OR) and corresponding 95\% confidence interval (CI); and (5) Study design: observational studies including cohort, case-control, or cross-sectional studies. No language restrictions were imposed.

Exclusion criteria included studies, animal experiments, case reports, reviews, expert opinions and editorials that did not provide sufficient data for analysis.

\section{Data extraction and quality assessment}

Two researchers (Li WQ and Zhang XJ) independently screened the literature, extracted data and evaluated the risk of bias of the included studies. If they had a difference of opinion, they would reach an agreement through a consultation or by discussing the matter with a third person. In our study, we used a homemade data extraction table to extract data, including the study source; number of years; type, location, and number of patients; number of patients with MC; age at the operation; age of onset; and significant risk factors and results.

This systematic review was based on a meta-analysis of epidemiological observational studies (MOOSE) [11]. We used the Newcastle-Ottawa Scale (NOS) to assess the methodological quality, risks of selection and cohort comparability bias, and outcomes of the included studies [12]. The score of this scale ranges from 0 to 9 points. Studies with NOS scores of 5 or more stars were considered high quality; otherwise, the quality of the study was considered poor, and these studies were excluded.

\section{Data analysis}

RevMan 5.3 statistical software and Stata 15.0 software were used for statistical analysis. The same potential predictors must have been studied at least twice in the analysis of the original data. For dichotomous results, the adjusted OR of the 95\% CI was calculated. For continuous results, the mean difference (MD) of the $95 \%$ CI was calculated.

We used Cochrane's Q statistics and $\mathrm{I}^{2}$ statistics to study heterogeneity. Heterogeneity was classified as low, medium, or high based on $\mathrm{I}^{2}$ values of $25,50 \%$, or $75 \%$, respectively [13]. If $P>0.1$ and $\mathrm{I}^{2} \leq 50 \%$, homogeneity was present and a fixed effect model was adopted; $P \leq$ 0.1 and $\mathrm{I}^{2}>50 \%$ indicated the presence of heterogeneity. We subsequently conducted a sensitivity analysis or subgroup analysis to explore possible explanations for the heterogeneity. For the predictors whose source of 
heterogeneity was not identified in the sensitivity or subgroup analysis, a random effect model was used in the meta-analysis. Publication bias was examined using Begg's test, with a $P$ value $<0.1$ indicating a significant difference.

\section{Results}

\section{Search results}

Four hundred fifty-eight articles were retrieved. First, 148 duplicate records were excluded. Second, 223 records were excluded after reading the titles and abstracts of the articles. Then, 62 records were excluded by reading the full texts. Finally, data from 3728 individual patients with 692 myasthenic crisis cases were included in the metaanalysis of 25 eligible studies [1, 7, 8, 14-36]. Figure 1 presents the literature search and study selection process.

\section{Basic characteristics of the included studies and risk of bias assessment}

Seven retrospective cohort studies and 18 case-control studies were included, and 14 risk factors for MCAT were extracted. The basic characteristics of the included studies are shown in Table 1 . The NOS criteria used to evaluate the quality of the 25 studies that were included in this meta-analysis are shown in Table 1 and Table 1 in the supplement. Significant risk factors associated with MCAT and the results of bias risk assessment are shown in Table 2.

\section{Risk factors}

Risk factors related to the patients' preoperative conditions (Fig. 2a)

Preoperative MC history Twelve studies $[8,20-23,27$, $29,31,33-36]$ provided data on the history of MC and included 2094 patients. The results of the heterogeneity test did not reveal heterogeneity among the studies $\left(\mathrm{I}^{2}=\right.$ $18 \%, P=0.26$ ); therefore, the fixed effect model was used for the meta-analysis. A preoperative history of $\mathrm{MC}$ was an independent risk factor for MCAT [OR $=4.13,95 \%$ CI $(3.08,5.54), P<0.00001]$.

Preoperative bulbar symptoms Eight studies [7, 16-18, $21,22,27,35]$ including 1443 patients reported the relationship between preoperative bulbar symptoms and MCAT. The results of the heterogeneity test did not reveal heterogeneity among the studies $\left(\mathrm{I}^{2}=7 \%, P=0.37\right)$, and thus the fixed effect model was used for the metaanalysis. Preoperative bulbar symptoms were an independent risk factor for MCAT $[\mathrm{OR}=3.71,95 \% \mathrm{CI}$ (2.54, 5.42), $P<0.00001]$.

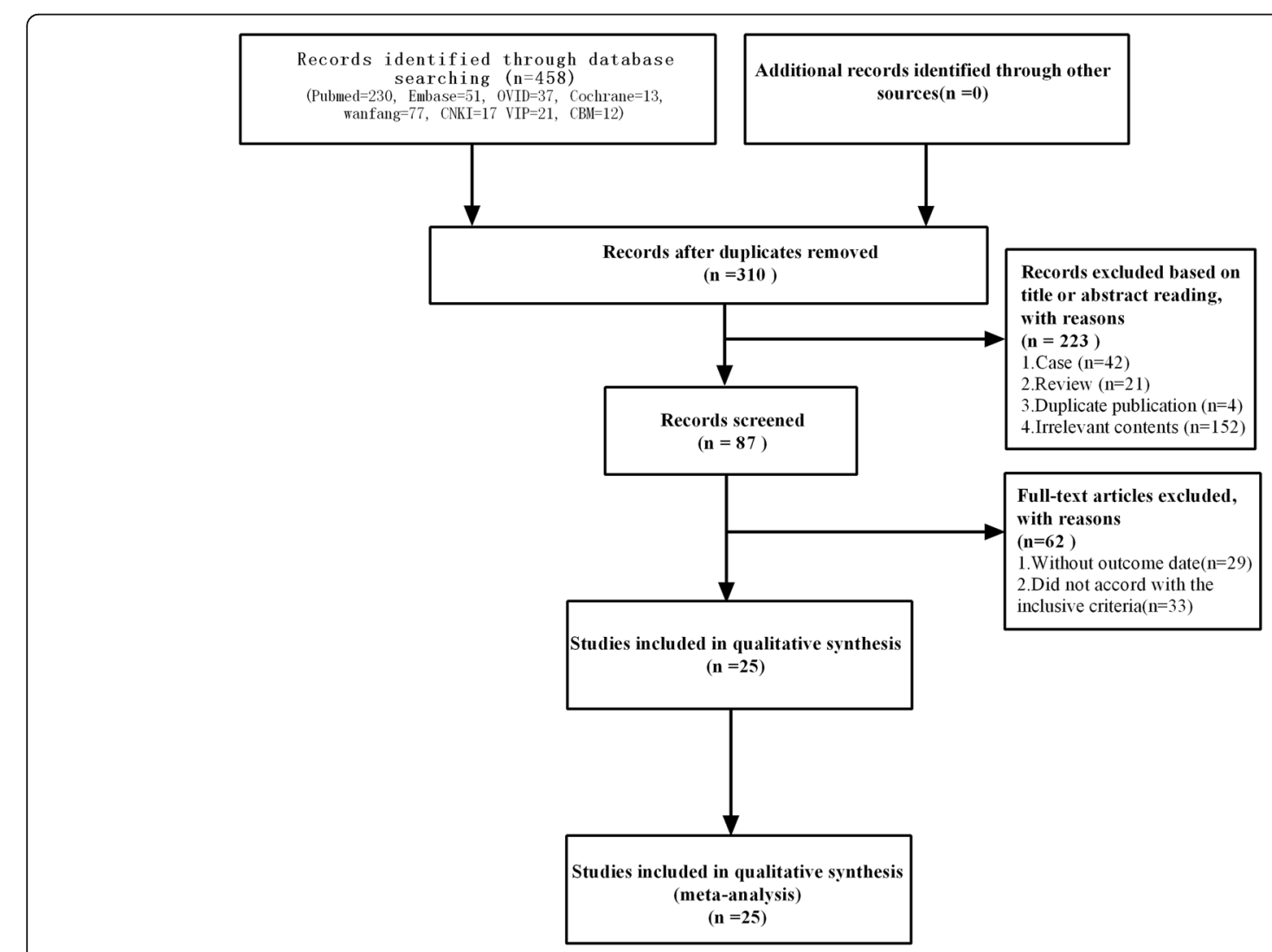

Fig. 1 Flow diagram of the literature search and selection process 


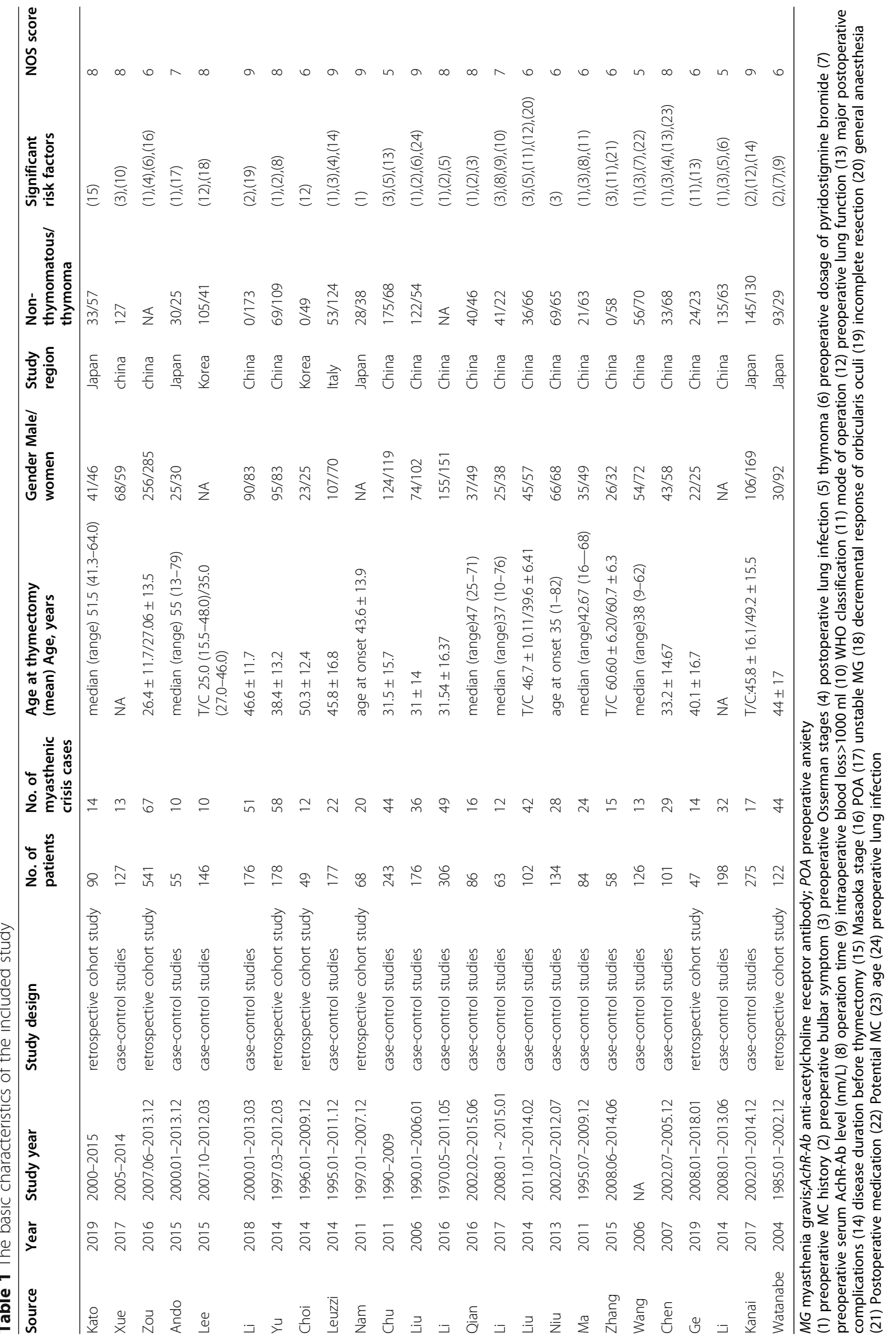


Liu et al. Journal of Cardiothoracic Surgery (2020) 15:270

Page 5 of 12

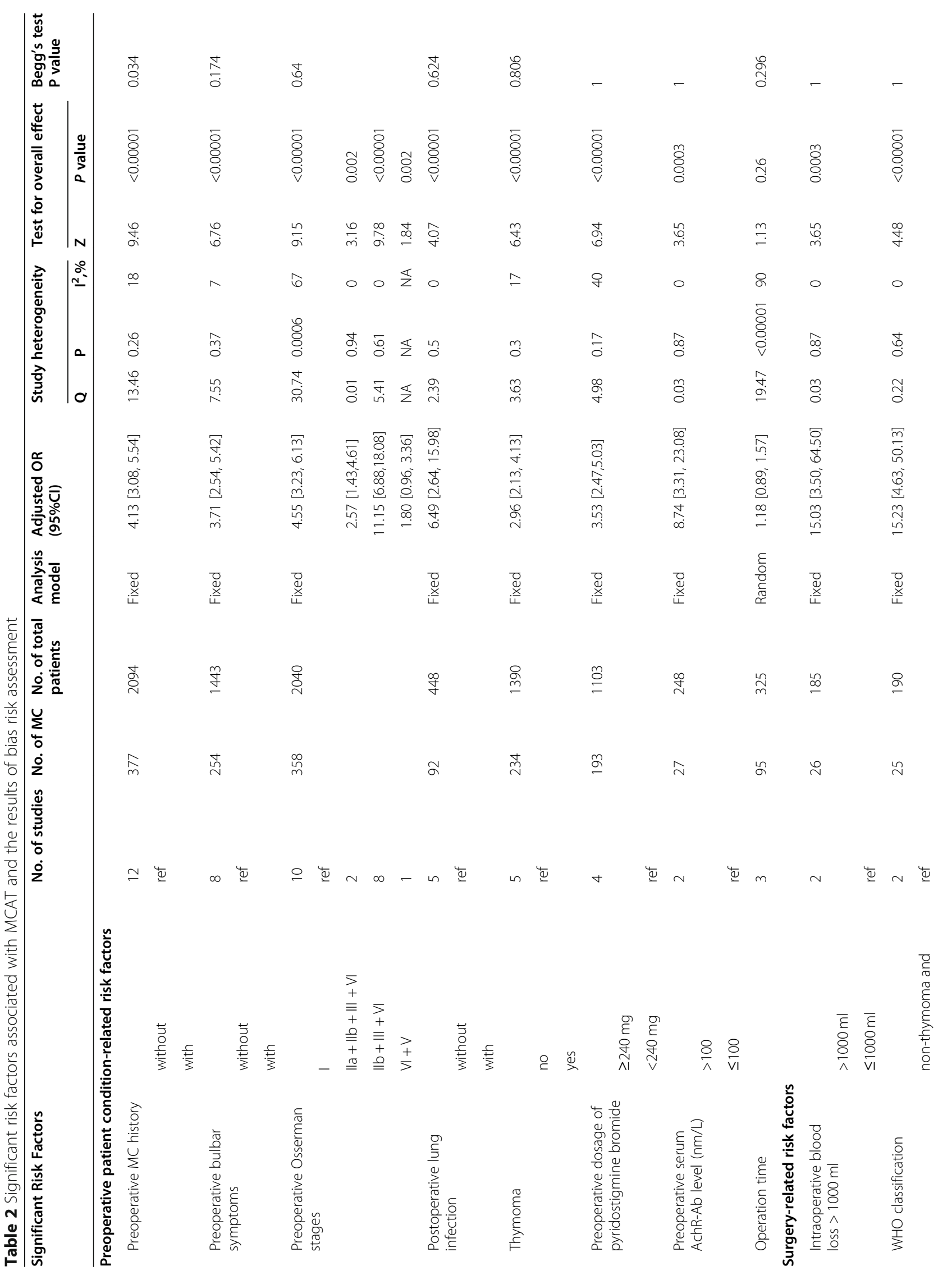


Lin et al. Journal of Cardiothoracic Surgery

(2020) $15: 270$

Page 6 of 12

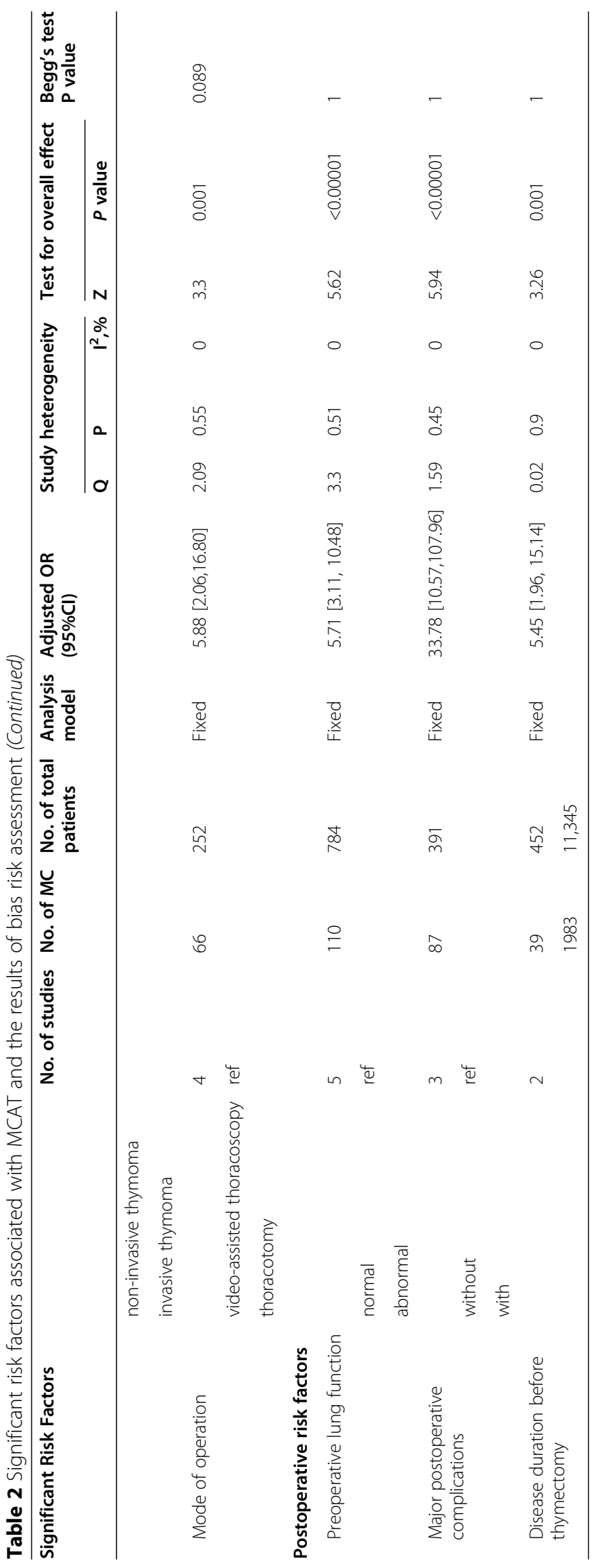




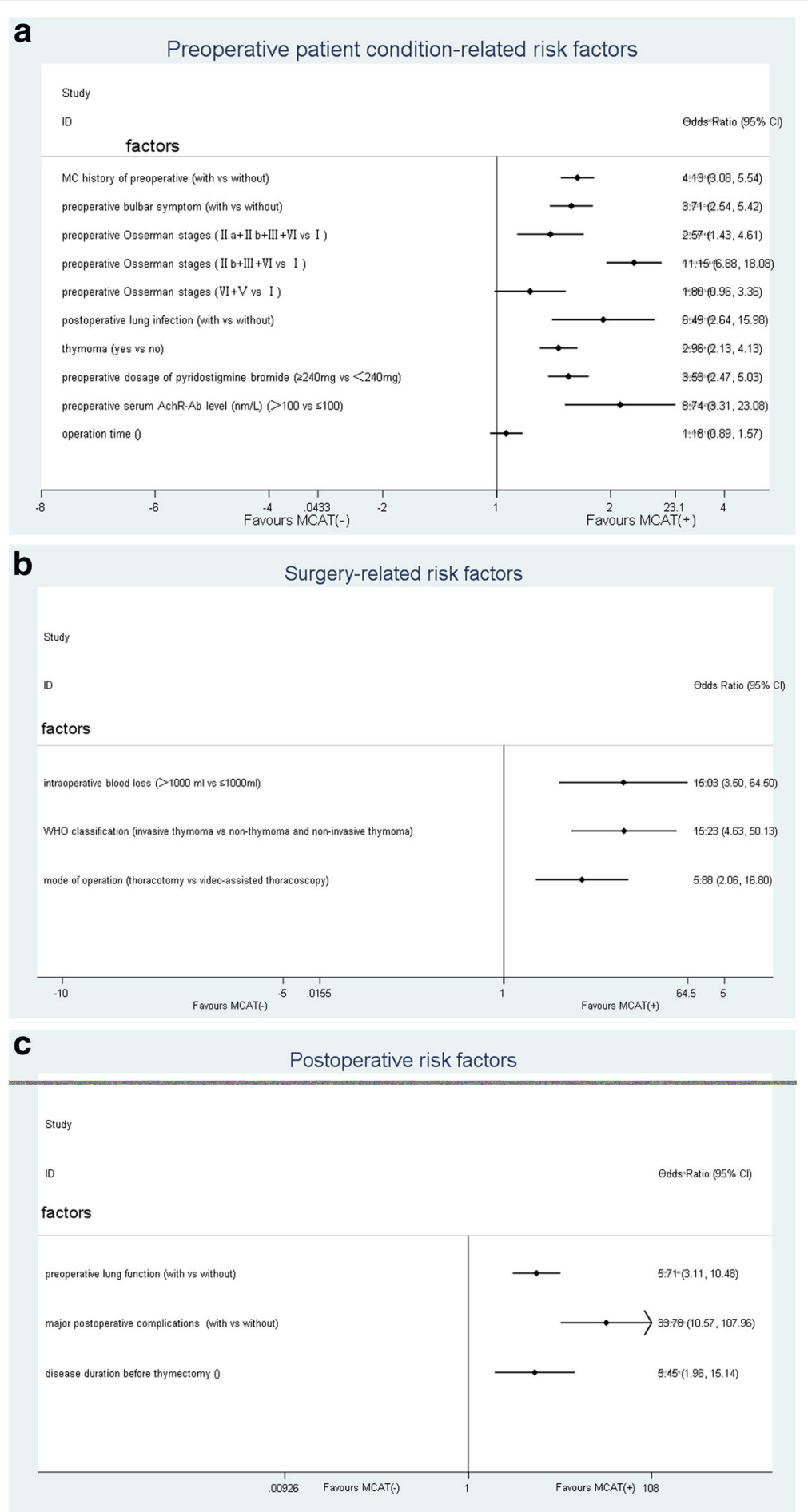

Fig. 2 a Meta-analyses of the association between MCAT and risk factors related to the patients' preoperative conditions. $\mathbf{b}$ Meta-analyses of the association between MCAT and surgery-related risk factors. c Meta-analyses of the association between MCAT and postoperative risk factors

Preoperative Osserman stages Ten studies $[8,19,20$, $22,28-30,32-34,36]$ including 2040 patients provided the relationship between the preoperative Osserman classification and MCAT. The results of the heterogeneity test showed evidence of heterogeneity between studies $\left(\mathrm{I}^{2}=67 \%, P=0.0006\right)$. According to the preoperative Osserman stages, the patients were divided into three subgroups: IIa + IIb + III + VI, IIb + III + VI, and $\mathrm{VI}+\mathrm{V}$. The results of the heterogeneity test did not reveal any heterogeneity $\left(\mathrm{I}^{2}=0, P>0.1\right)$; therefore, the 
fixed effect model was used for the analysis. Notably, $\mathrm{IIa}+\mathrm{IIb}+\mathrm{III}+\mathrm{VI}[\mathrm{OR}=2.57,95 \%$ CI $(1.43,4.61), P=$ $0.002], \quad \mathrm{IIb}+\mathrm{III}+\mathrm{VI}[\mathrm{OR}=11.15,95 \% \mathrm{CI}(6.88,18.08)$, $P<0.00001]$, and VI $+\mathrm{V}[\mathrm{OR}=1.80,95 \% \mathrm{CI}(0.96,3.36)$, $P=0.07]$ were all statistically significant. Based on the analysis described above, preoperative Osserman stages were an independent risk factor for MCAT, and patients with preoperative type IIb + III + VI stages had the highest risk of MCAT.

Preoperative dosage of pyridostigmine bromide Five studies [20, 22, 28, 29, 35] including 1103 patients provided data on the relationship between the preoperative pyridostigmine bromide dosage and MCAT. The results of the heterogeneity test showed evidence of heterogeneity between studies $\left(\mathrm{I}^{2}=92 \%, P<0.00001\right)$. After the sensitivity analysis, the study by Liu et al. [28] may have been the source of heterogeneity, and the heterogeneity test was repeated after excluding this study. No heterogeneity was observed between the studies $\left(\mathrm{I}^{2}=40 \%, P=\right.$ $0.17)$, which were analysed using a fixed effect model. Moreover, the dosage of pyridostigmine bromide before the operation was an independent risk factor for MCAT $[\mathrm{OR}=3.53,95 \% \mathrm{CI}(2.47,5.03), P<0.00001]$.

Preoperative serum acetylcholine receptor antibody (AchR-Ab) level Two studies [7, 36] reported the relationship between the preoperative AchR-Ab level and MCAT, including a total of 248 patients. The results of the heterogeneity test showed no evidence of heterogeneity between the studies $\left(\mathrm{I}^{2}=0 \%, P=0.89\right)$, and thus the fixed effect model was used for the metaanalysis. A preoperative AchR-Ab level $>100 \mathrm{~nm} / \mathrm{L}$ was an independent risk factor for MCAT $[\mathrm{OR}=8.74$, 95\% CI $(3.31,23.08), P<0.00001]$.

Preoperative lung function Five studies $[17,18,22,24$, $28]$ including 784 patients reported the relationship between preoperative lung function and MCAT. The results of the heterogeneity test did not reveal heterogeneity among the studies $\left(\mathrm{I}^{2}=0 \%, P=0.51\right)$; therefore, the fixed effect model was used for the metaanalysis. The risk of MCAT in patients with abnormal preoperative lung function was 5.71 times higher than for patients with a normal preoperative pulmonary function. Preoperative pulmonary function was an independent risk factor for MCAT [OR $=5.71,95 \%$ CI (3.11, 10.48), $P<0.00001]$.

Major preoperative complications Three studies [15, 32, 34] including 391 patients reported the relationship between preoperative complications and MCAT. The results of the heterogeneity test did not detect heterogeneity between the studies $\left(\mathrm{I}^{2}=0 \%, P=0.45\right)$, and thus the fixed effect model was used for the meta-analysis. The risk of MCAT in patients with preoperative complications was 33.78 times higher than in patients without complications. Preoperative complications were independent risk factors for MCAT $[\mathrm{OR}=33.78,95 \% \mathrm{CI}$ (10.57, 107.96), $P<0.00001]$.

Disease duration before thymectomy Two studies [8, 18] including 452 patients reported the relationship between the preoperative disease duration before thymectomy and MCAT. The results of the heterogeneity test showed no heterogeneity between the studies $\left(\mathrm{I}^{2}=0 \%\right.$, $P=0.90$ ), and thus the fixed effect model was used for the meta-analysis. The preoperative course of disease was an independent risk factor for MCAT $[\mathrm{OR}=5.45$, 95\% CI $(1.96,15.14), P=0.001]$. According to Kanai et al. [18], a disease duration $<3$ months was a risk factor for MCAT $[\mathrm{OR}=5.18,95 \%$ CI $(1.42,18.8)]$. Leuzzi et al. [8] identified a disease course of $>2$ years as a risk factor for MCAT $[\mathrm{OR}=5.94,95 \%$ CI $(1.12,31.48)]$.

\section{Surgery-related risk factors (Fig. 2b)}

Operation time Three studies $[19,27,33]$ that included 325 patients provided data on the relationship between the operation time and MCAT. The results of the heterogeneity test showed substantial heterogeneity between studies $\left(\mathrm{I}^{2}=90 \%, P<0.00001\right)$. The random effect model was used for the analysis. The operation time was not an independent risk factor for MCAT $[\mathrm{OR}=1.18$, $95 \%$ CI $(0.89,1.57), P=0.26]$.

Intraoperative blood loss $>1000 \mathbf{~ m L}$ Two studies [7, 19] reported the relationship between intraoperative blood loss and MCAT and included 185 patients. The results of the heterogeneity test showed no heterogeneity between the studies $\mathrm{I}^{2}=0 \%, P=0.87$ ); therefore, the fixed effect model was used for the analysis. The risk of MCAT in patients with intraoperative blood loss $>1000$ $\mathrm{mL}$ was 15.03 times greater than patients with an intraoperative blood loss $\leq 1000 \mathrm{~mL}$. Intraoperative blood loss $>1000 \mathrm{~mL}$ was an independent risk factor for MCAT $[\mathrm{OR}=15.03,95 \% \mathrm{CI}(3.50,64.50), P=0.0003]$.

Mode of operation Four studies $[15,19,25,33]$ reported the relationship between the mode of operation and postoperative MC and included 252 patients. The results of the heterogeneity test did not identify heterogeneity among the studies $\left(\mathrm{I}^{2}=0 \%, P=0.55\right)$, and thus the fixed effect model was used for the meta-analysis. The risk of MCAT after thoracotomy was 5.88 times greater than after video-assisted thoracoscopy. The mode of operation was an independent risk factor for MCAT $[\mathrm{OR}=5.88,95 \% \mathrm{CI}(2.06,16.80), P=0.0010]$. 


\section{Postoperative risk factors (Fig. 2c)}

Postoperative lung infection Five studies $[8,20,22,33$, 34] including a total of 989 patients reported the relationship between postoperative lung infection and MCAT. The results of the heterogeneity test showed no evidence of heterogeneity between the studies $\left(\mathrm{I}^{2}=\right.$ $0 \%, P=0.45)$; therefore, the fixed effect model was used for the analysis. A postoperative lung infection was an independent risk factor for MCAT $[\mathrm{OR}=4.43$, 95\% CI $(2.38,8.22), P<0.00001]$.

Thymoma Five studies $[20,21,28,29,32]$ provided data on the relationship between thymoma and MCAT, including 1390 patients. The results of the heterogeneity test showed evidence of heterogeneity between studies $\left(\mathrm{I}^{2}=79 \%, P=0.0008\right)$. After the sensitivity analysis, the study by Chu et al. [32] may have been the source of heterogeneity, and the heterogeneity test was repeated after excluding this study. No heterogeneity was observed between the studies $\left(\mathrm{I}^{2}=17 \%, P=0.30\right)$, which were analysed using a fixed effect model. Thymoma was an independent risk factor for MCAT [OR $=2.96,95 \%$ CI $(2.13,4.13), P<0.00001]$.

World Health Organization (WHO) classification Two studies $[17,19]$ reported the relationship between the postoperative thymic WHO classification and MCAT. The results of the heterogeneity test did not reveal heterogeneity between the studies $\left(\mathrm{I}^{2}=0 \%, P=\right.$ 0.64), and thus the fixed effect model was used for the meta-analysis. The risk of MC in patients with invasive thymoma was 15.23 times greater than in patients without thymoma and patients with noninvasive thymoma. The postoperative WHO classification was an independent risk factor for MCAT $[\mathrm{OR}=15.23,95 \%$ CI (4.63, 50.13), $P<0.00001$ ].

\section{Discussion}

\section{Main findings and interpretation of the results}

The purpose of this meta-analysis is to provide evidence for the predictors of MCAT. An understanding of the risk factors for MCAT is helpful for doctors and patients to coordinate the optimal postoperative management strategy. Overall, 25 studies in our meta-analysis reported risk factors for MCAT based on an analysis of 11,345 patients, and the incidence of MCAT complications was $17.48 \%$. The results of the meta-analysis identified the following factors related to the condition of patients before the operation as risk factors for MC: a history of preoperative MG, preoperative bulbar symptoms, preoperative Osserman stages, preoperative dosage of pyridostigmine bromide, preoperative serum acetylcholine receptor antibody (AchR-Ab) level $(>100 \mathrm{~nm} / \mathrm{L})$, preoperative lung function, major postoperative complications, and disease duration before thymectomy. Surgery-related risk factors were blood loss $>1000 \mathrm{~mL}$ and the mode of operation (thoracotomy). Postoperative risk factors were postoperative lung infection, thymoma, and the WHO classification.

A history of preoperative MG, preoperative bulbar symptoms, preoperative Osserman stages, and preoperative lung function were risk factors for postoperative $\mathrm{MC}$, consistent with the results reported by AKaishi et al. [37] regarding the preoperative predictors of MCAT.

Preoperative Osserman stages reflect the severity of MG symptoms; type I is limited to extraocular muscles, type II and above include the involvement of other skeletal muscles, and patients with type IIb and above have symptoms of medulla oblongata muscle involvement, such as swallowing difficulty. Most of the studies [30, 32] reported that the preoperative Osserman stage was an independent factor affecting the occurrence of MCAT. Xue et al. [17] proposed that the WHO histological classification B2-B3 and Osserman stages IIa-IV independently predicted preoperative risks of a postoperative myasthenic crisis (POMC).

As shown in the study by Lucchi et al. [38], compared with type (I + IIa), type (IIb + III + IV) had a worse prognosis, which was related to the involvement of swallowing muscles before the operation. The potential explanations for the relationship between preoperative Osserman stages and the prognosis are listed below. First, type I and IIa symptoms are mainly concentrated in the ocular muscles and affect limb muscle strength, but do not involve the respiratory muscles. The symptoms associated with type IIb and above involve respiratory muscles, and patients with MG are prone to dyspnoea. Second, patients with type IIb and greater symptoms present with abnormal mastication and swallowing functions, which can easily cause coughing and choking while drinking water or the accumulation of saliva that is unable to swallowed and mistakenly passes into the airway, resulting in respiratory tract obstruction and crisis.

A history of preoperative $\mathrm{MC}$ and bulbar symptoms were identified as independent risk factors for developing POMC, which represented the severe status of MG. Additionally, in patients with systemic MG, the disease should be controlled to the greatest extent possible before the operation to reduce the preoperative stages, which is helpful to reduce the incidence of MCAT and modulate the long-term effect.

Few studies $[7,36]$ examined the correlation between the preoperative AchR-Ab titre and MCAT. The result of the meta-analysis showed that a high serum AchR-Ab level (AchR-Ab level $>100 \mathrm{~nm} / \mathrm{L}$ ) was related to the occurrence of MCAT. 
The disease duration before thymectomy was an independent factor affecting the occurrence of MCAT in the present study. According to Leuzzi et al. [8], a symptom duration of $>2$ years independently predicted POMC. A longer duration of MG was reported to contribute to the risk of a postoperative $\mathrm{MC}[8,39]$. A long disease duration is generally related to a poor treatment response [40], probably due to the cumulative damage at the neuromuscular junction.

In contrast, some reports [7, 27] revealed a tendency for a short disease duration to be related to the risk of a postoperative MC. Kanai et al. [18] identified a disease duration of $<3$ months as a risk factor for POMC. The short course of the disease may reflect the rapid progression of MG or an insufficient response to treatment designed to inhibit the activity of the disease. The specific mechanism of $\mathrm{MC}$ and the course of the disease require further analysis.

Few reports have analysed the effect of MG associated with immune diseases on MCAT. Chen et al. [34] reported that concomitant immune disease (hyperthyroidism) was an important factor predicting the occurrence of postoperative MC. For patients with MG complicated with other autoimmune diseases, as indicated by a preoperative examination, close observation and early prevention measures should be implemented after the operation, which may reduce the possibility of postoperative MC to a certain extent. However, due to the inclusion of a limited number of cases, further studies are needed to determine and verify whether other autoimmune diseases are risk factors for long-term effects in later stages.

Thymectomy in patients with MG is divided into traditional thoracotomy and thoracoscopic surgery. According to our meta-analysis, thoracotomy was an independent risk factor for MCAT. This factor may be related to the trauma and bleeding associated with traditional thoracotomy, which easily causes postoperative pulmonary infection. Watanabe et al. [7] showed that intraoperative blood loss $>1000 \mathrm{~mL}$ was a prognostic factor for postoperative MC. The risk of MCAT in patients with intraoperative blood loss $>1000 \mathrm{~mL}$ was 18.52 times greater than that in patients with an intraoperative blood loss $\leq 1000 \mathrm{~mL}$. The mechanism of intraoperative blood loss $>1000 \mathrm{~mL}$ affecting postoperative myasthenia crisis is unclear.

The dosage of pyridostigmine bromide before the operation is an independent risk factor for MCAT. An acetylcholinesterase inhibitor (AChEI) increases the functional concentration of acetylcholine by blocking acetylcholinesterase at the neuromuscular junction to improve muscle contraction, strength and function. The preoperative use of large doses of cholinesterase inhibitors will activate muscarinic receptors, accelerate the destruction of postsynaptic acetylcholine receptors (AChRs) at the neuromuscular junction, decrease the ability of patients to cough up sputum due to postoperative pain, cause excessive respiratory secretions after the operation, increase the risk of respiratory tract infection, and increase the risk of crisis.

The meta-analysis identified a postoperative pulmonary infection as the main risk factor for MCAT in patients with MG. Pulmonary infection increases respiratory secretions and reduces the area of the effective alveolar membrane, which leads to MCAT. However, a postoperative infection does not indicate that the $\mathrm{MC}$ lasts for a long time [41].

Pulmonary function tests directly evaluate the function of respiratory muscles and reflect the compliance of respiratory muscles. Prigent et al. [42] reported the therapeutic effect on ICU patients and the timing of endotracheal intubation and extubation in MG patients based on measurements of vital capacity (VC).

Preoperative pulmonary ventilation function plays an important role in predicting MCAT. Pulmonary function tests directly evaluate the function and reflect the compliance of respiratory muscles. Weakness of these muscles can be evaluated by the forced vital capacity (FVC), and these measurements are useful to detect respiratory muscle failure in MG [42]. As shown in the study by Loach et al. [43], patients with MG presenting a preoperative $\mathrm{VC}>2 \mathrm{~L}$ were less likely to experience a postoperative crisis. Choi et al. [26] showed that the optimal cut-off point of preoperative pulmonary function to determine the risk of MCAT was $65 \%$ of the mean FVC (mFVC)/peak FVC (pFVC), indicating that patients with a significantly reduced $\mathrm{mFVC} / \mathrm{pFVC}$ value may not be candidates for thymectomy. Thymectomy is not suitable for patients with MG presenting poor pulmonary function but may be considered after stabilization, and these patients should be closely monitored during the postoperative period.

The relationship between thymoma and postoperative crisis has been controversial [44, 45]. The meta-analysis identified thymoma as an independent risk factor for MCAT, and pathological types (invasive thymoma, B2B3) were independent risk factors for MCAT. A potential explanation for this finding is that invasive thymoma is generally so invasive that it is difficult to remove during the operation, and thus the tumour (particularly type B thymoma) has a high probability of metastasis.

The meta-analysis did not identify the operation time as an independent risk factor for MCAT. Nevertheless, we are unable to exclude this variable as a potential risk factor, as some studies [19, 27, 33] have reported a significant association of the operation time with MCAT. The results of this study exhibited substantial heterogeneity. Because the original data were unable to be 
obtained, a subgroup analysis was not conducted to explore the source of its heterogeneity.

\section{Implications from this meta-analysis Limitations}

Our research has several limitations that should be explained. First, because the studies we included are retrospective cohort studies and retrospective case-control studies and the related risk factors for MCAT are diverse and complex, these factors are affected by selection bias to some extent. Randomized controlled trials are difficult to perform, and selection bias caused by researchers and patients in clinical studies is inevitable. Second, some of the included research samples are relatively small, which once again indicates the need for further high-quality, large cohort studies in the future. In addition, the definition of MCAT is not uniform, the follow-up time is not consistent, and the outcome may thus be affected to some extent. Finally, individual studies lack detailed data, and consequently, no further subgroup analyses were able to be conducted to explore the sources of heterogeneity.

\section{Conclusions}

This study combines the most recent evidence of risk factors for MCAT in patients with MG. The results of this meta-analysis confirm that the pathogenesis of MCAT is unclear and many risk factors for MCAT exist. The independent risk factors for MCAT were a preoperative history of $\mathrm{MC}$, preoperative bulbar symptoms, preoperative MG Osserman stages, preoperative dosage of pyridostigmine bromide, preoperative serum AchRAb level (> $100 \mathrm{~nm} / \mathrm{L})$, lung function, major postoperative complications, disease duration before thymectomy, blood loss $>1000 \mathrm{~mL}$, thoracotomy, postoperative lung infection, thymoma, and WHO classification.

\section{Supplementary information}

Supplementary information accompanies this paper at https://doi.org/10 1186/s13019-020-01320-x.

Additional file 1: Table S1. NOS criteria were used to evaluate the quality of the 25 included studies.

\section{Abbreviations \\ MG: Myasthenia gravis; MCAT: myasthenic crisis after thymectomy; AchR- Ab: anti-acetylcholine receptor antibody; MC: MG crisis; NOS: Newcastle- Ottawa Scale; MD: Mean difference; WHO: World Health Organization; VC: Vital capacity; POA: preoperative anxiety}

\section{Acknowledgments}

Not applicable.

\section{Authors' contributions}

CY Liu designed the research; WQ Li and XJ Zhang independently screened the literature, extracted data and evaluated the risk of bias of the included studies. P Liu made important contributions to data extraction and analysis;
CY Liu wrote the manuscript; GY Qi revised the manuscript. All authors approved the final version of the manuscript.

Funding

This study was financially supported by grants from the Shijiazhuang Science and Technology Bureau Foundation (Grant no. 171461553), Key Research of Medical Science Research of Hebei Province, China (Grant no. 20190165), and Special Project of Beijing-Tianjin-Hebei Basic Research Cooperation (Grant no. H2019106063).

\section{Availability of data and materials}

The datasets generated and analysed are available from the corresponding author on reasonable request.

Ethics approval and consent to participate

Not applicable.

\section{Consent for publication}

Not applicable.

\section{Competing interests}

The authors declare no conflicts of interest.

Received: 17 June 2020 Accepted: 21 September 2020

Published online: 29 September 2020

\section{References}

1. Qi G, Xue Y, Li Y, et al. Docetaxel/cisplatin Therapy in Myasthenia Gravis with Hypertension/diabetes. Open Med (Wars). 2017;12:403-8.

2. Richman DP, Agius MA. Treatment of autoimmune myasthenia gravis. Neurology. 2003;61:1652-61.

3. Blalock A, Mason MF, Morgan HJ, et al. Myasthenia Gravis and tumors of the thymic region:report of a case in which the tumor was removed. Ann Surg. 1939;110:544-61.

4. Blalock A, Harvey AM, Ford FR, et al. The treatment of myasthenia gravis by removal of the thymus gland:preliminary report. J Am Med Assoc. 1941;117: 1529-33.

5. Kas J, Kiss D, Simon V, et al. Decade-long experience with surgical therapy of myasthenia gravis: early complications of 324 transsternal thymectomies. Ann Thorac Surg. 2001;72:1691-7.

6. Prokakis C, Koletsis E, Salakou S, et al. Modified maximal thymectomy for myasthenia gravis:effect of maximal resection on late neurologic outcome and predictors of disease remission. Ann Thorac Surg. 2009;88:1638-45.

7. Watanabe A, Watanabe T, Obama T, et al. Prognostic factors for myasthenic crisis after transsternal thymectomy in patients with myasthenia gravis. J Thorac Cardiovasc Surg. 2004:127:868-76.

8. Leuzzi G, Meacci E, Cusumano G, et al. Thymectomy in myasthenia gravis: proposal for a predictive score of postoperative myasthenic crisis. Eur J Cardiothorac Surg. 2014;45:e76-88 e88.

9. Sanders DB, Wolfe Gl, Benatar M, et al. International consensus guidance for management of myasthenia gravis: executive summary. Neurology. 2016;87: 419-25.

10. Geng $Y$, Zhang $H$, Wang $Y$. Risk factors of myasthenia crisis after thymectomy among myasthenia gravis patients. Medicine. 2020;99:e18622.

11. Stroup DF, Berlin JA, Morton SC, et al. Meta-analysis of observational studies in epidemiology:a proposal for reporting. Meta-analysis of Observational Studies in Epidemiology (MOOSE) group. JAMA. 2000;283:2008-12.

12. Stang A. Critical evaluation of the Newcastle-Ottawa scale for the assessment of the quality of nonrandomized studies in meta-analyses. Eur J Cardiothorac Surg. 2010;25:603-5

13. Higgins JP, Thompson SG, Deeks JJ, et al. Measuring inconsistency in metaanalyses. BMJ. 2003;327:557-60.

14. Kato T, Kawaguchi K, Fukui T, et al. Risk factors for the exacerbation of myasthenic symptoms after surgical therapy for myasthenia gravis and thymoma. Semin Thorac Cardiovasc Surg. 2020;32(2):378-85.

15. Ge XQ. Analysis of the occurrence and long-term rffect of postoperative myasthenia gravis crisis in patients with myasthenia gravis compllicated with abnormal thymus. Guangxi Med Univ. 2019;9:15-9.

16. Li Y, Wang $H$, Chen $P$, et al. Clinical outcome and predictive factors of postoperative myasthenic crisis in 173 thymomatous myasthenia gravis patients. Int J Neurosci. 2018;128:103-9. 
17. Xue L, Wang L, Dong J, et al. Risk factors of myasthenic crisis after thymectomy for thymoma patients with myasthenia gravis. Eur J Cardiothorac Surg. 2017;52:692-7.

18. Kanai T, Uzawa A, Sato Y, et al. A clinical predictive score for postoperative myasthenic crisis. Ann Neurol. 2017;82:841-9.

19. Li YF, Li YK, Wang YZ, Cheng Q, Zheng Z, Liu JP. Analysis of risk factors of myasthenia gravis crisis in myasthenia gravis patients during perioperative period for thymectomy. J Clin Neurol. 2017;30:16-9.

20. Zou J, Su C, Lun X, et al. Preoperative anxiety in patients with myasthenia gravis and risk for myasthenic crisis after extended transsternal thymectomy: a consort study. Medicine (United States). 2016;95(10):e2828.

21. Li J, Shang K. Analysis of risk factors of myasthenia crisis after thymectomy in patients with myasthenia gravis. J Int Intensive Med. 2016;22:297-9.

22. Qian KJ. Risk factors and scoring model establishment of myasthenic crisis after thymectomy for patients with myasthenia gravis. Xinjiang Med Univ. 2016:9:24-6.

23. Ando T, Omasa M, Kondo T, et al. Predictive factors of myasthenic crisis after extended thymectomy for patients with myasthenia gravis. Eur J Cardiothorac Surg. 2015:48:705-9,709.

24. Lee HS, Lee HS, Lee HE, et al. Predictive factors for myasthenic crisis after videoscopic thymectomy in patients with myasthenia gravis. Muscle Nerve. 2015;52:216-20.

25. Zhang ZF, Jia R, Cui YB, et al. Risk factors for postoperative myasthenia crisis in elderly patients with delayed myasthenia gravis. Chin J Gerontol. 2015;35: 6210-1.

26. Choi KH, Nam TS, Lee SH, et al. Preoperative pulmonary function is strongly related to myasthenic crisis after thymectomy. Neurol India. 2014;62:164-8.

27. Yu S, Lin J, Fu X, et al. Risk factors of myasthenic crisis after thymectomy in 178 generalized myasthenia gravis patients in a five-year follow-up study. Int J Neurosci. 2014;124:792-8.

28. Liu XQ, Sang L, Chen $S B$, et al. Analysis of crisis after thymectomy in patients with myasthenia gravis. Guangdong Med J. 2014;35:3667-9.

29. Li F. Risk factors of postoperative Myasthenic crisis in patients with myasthenia gravis. Occup Health. 2014;30:718-21.

30. Niu YD, Wang TS, Zhao S, Ding LX. Predictors of early postoperative crisis for myasthenia gravis. Chin J Gerontol. 2013;33:2362-4.

31. Nam TS, Lee SH, Kim BC, et al. Clinical characteristics and predictive factors of myasthenic crisis after thymectomy. J Clin Neurosci. 2011;18: $1185-8$.

32. Chu XY, Xue ZQ, Wang RW, et al. Predictors of postoperative myasthenic crisis in patients with myasthenia gravis after thymectomy. Chin Med J (Engl). 2011;124:1246-50

33. Ma JS, Wang XL, Chen K, et al. Factors contributing to myasthenic crisis after thymectomy-a multivariate analysis study. Chin J Thorac Cardiocasc Surg. 2011;27:27-9.

34. Chen J, Pang LW, Chen ZM, et al. Factors contributing to Myasthenic crisis after Thymectomy-a multivariate analysis study. Chin J Clin Neurosci. 2007; 15:169-73.

35. Liu WB, Men LN, Tang BY, et al. Prognostic factors of myasthenic crisis after extended thymectomy in patients with generalized myasthenia gravis. Zhonghua Yi Xue Za Zhi. 2006;86:2737-40.

36. Wang QG, Yang KP, Shou HS, Ding XQ, Guo L. Risk factors of myasthenia gravis crisis after thymectomy. Chin J Thorac Cardiovasc Surg. 2006;22:2689.

37. Akaishi T, Motomura M, Shiraishi $\mathrm{H}$, et al. Preoperative risks of post-operative myasthenic crisis (POMC): a meta-analysis. J Neurol Sci. 2019;407:116530.

38. Lucchi M, Ricciardi R, Melfi F, et al. Association of thymoma and myasthenia gravis:oncological and neurological results of the surgical treatment. Eur J Cardiothorac Surg. 2009:35:812-6 816

39. Leventhal SR, Orkin FK, Hirsh RA. Prediction of the need for postoperative mechanical ventilation in myasthenia gravis. Anesthesiology. 1980;53:26-30.

40. Tellez-Zenteno JF, Remes-Troche JM, Garcia-Ramos G, et al. Prognostic factors of thymectomy in patients with myasthenia gravis: a cohort of 132 patients. Eur Neurol. 2001;46:171-7.

41. Krawczyk P, Adamczyk-Korbel M, Kieszko R, et al. Immunological system status and the appearance of respiratory system disturbances in thymectomized patients. Arch Immunol Ther Exp (Warsz). 2007:55:49-56.

42. Prigent $H$, Orlikowski D, Letilly N, et al. Vital capacity versus maximal inspiratory pressure in patients with Guillain-Barre syndrome and myasthenia gravis. Neurocrit Care. 2012;17:236-9.
43. Loach L, Davis JN, Lange MJ, et al. Postoperative management after Thymectomy. Br Med J. 1975:2:37.

44. Tansel T, Onursal E, Barlas $S$, et al. Results of surgical treatment for nonthymomatous myasthenia gravis. Surg Today. 2003;33:666-70.

45. Bachmann K, Burkhardt D, Schreiter I, et al. Long-term outcome and quality of life after open and thoracoscopic thymectomy for myasthenia gravis: analysis of 131 patients. Surg Endosc. 2008;22:2470-7.

\section{Publisher's Note}

Springer Nature remains neutral with regard to jurisdictional claims in published maps and institutional affiliations.
Ready to submit your research? Choose BMC and benefit from:

- fast, convenient online submission

- thorough peer review by experienced researchers in your field

- rapid publication on acceptance

- support for research data, including large and complex data types

- gold Open Access which fosters wider collaboration and increased citations

- maximum visibility for your research: over $100 \mathrm{M}$ website views per year

At $\mathrm{BMC}$, research is always in progress.

Learn more biomedcentral.com/submissions 